\title{
Die Prozessstruktur von teilautonomen Schulen und ihre Effektivität für die Herstellung optimaler Lernkontexte für schulische Bildungsprozesse
}

\section{Katharina Maag Merki und Brigitte Steinert}

Die Befunde zur Effektivität von teilautonomen Schulen sind nicht nur inkonsistent, sondern auch unzureichend. Insbesondere fehlen Studien, die teilautonome Schulen in ihrem Implementierungsgrad differenziert untersuchen. In der vorliegenden explorativen Studie wird der Frage nachgegangen, inwiefern sich der Grad der Umsetzung von Teilautonomie in Gymnasien in den Wahrnehmungen der Schüler/innen zu Schule und Unterricht abbildet. Die Ergebnisse zeigen, dass die Schüler/innen in Schulen mit einem hohen Grad an Kooperation zwischen Lehrpersonen in einem systematisch stärkeren Ausmass eine unterstützende, selbstaktivierende und reflexive Unterrichtsgestaltung und ein positives Schulklima wahrnehmen als Schüler/innen in Schulen mit einem geringen Ausmass an Kooperation zwischen Lehrpersonen.

Aus internationaler Perspektive kann die Einrichtung von teilautonomen Schulen als Megatrend der aktuellen Schulentwicklung bezeichnet werden (Burkard $\&$ Eikenbusch, 2002). Schulen bzw. die Lehrpersonen erhalten damit in einzelnen Teilen ihres beruflichen Handelns mehr schulische Gestaltungsmöglichkeiten. Dadurch wird die Erwartung verknüpft, dass die Schulen eine optimale Lehr-/Lernnumgebung entwickeln können, welche zu bestmöglichen Ergebnissen und Leistungen bei den Schüler/innen führt.

Wie auf der Basis einer ländervergleichenden Analyse gezeigt worden ist, kann Teilautonomie in verschiedenen Bereichen (Unterricht, Administration/ Organisation, Finanzen, Personalführung) gewährt werden (Organisation für wirtschaftliche Zusammenarbeit und Entwicklung [OECD],1995). Werden die verschiedenen Bildungssysteme innerhalb der Schweiz oder Deutschland und im internationalen Kontext verglichen, so zeigen sich nicht nur systematische Unterschiede in der inhaltlichen Ausgestaltung der Teilautonomie, sondern auch im Ausmass der Entscheidungsspielräume (Avenarius, Kimmig \& Rürup, 2003; Egloff \& Zahner Rossier, 2005; OECD, 2004). In der deutschen Schweiz ist die Gewährung von schulischer Teilautonomie systematisch verknüpft mit der Implementation von Schulleitungen, die Aufgaben im Bereich der pädagogischen und administrativen Führung der Schule zu übernehmen haben. Diese beiden 
Reformen sind Teil eines neuen Steuerungskonzeptes (Fend, 2005), das die Zuweisung erweiterter Handlungs- und Entscheidungsmöglichkeiten kombiniert mit der Verpflichtung der Einzelschule auf Qualitätsentwicklung und Rechenschaftslegung und der Implementierung eines systematischen Monitoringsystems auf System- und Einzelschulebene. Wie auf internationaler Ebene stellt die Teilautonomisierung von Schulen einzig ein Element im Kontext anderer Reformmassnahmen dar (z.B. zentrale Abschlussprüfungen, Einführung von Bildungsstandards).

Wie sieht die empirische Bilanz der Effektivität von teilautonomen Schulen aus? Hier stellt sich die Frage, an welchen Kriterien diese Effektivität gemessen werden soll. Das wichtigste Kriterium bezieht sich auf die Förderung der Schüler/innen in ihren fachlichen und überfachlichen Kompetenzen und der Herstellung eines optimalen Lernkontextes. Wie Leithwood und Menzies (1998) in einer Metaanalyse zwischen 1985 und 1995 im angloamerikanischen Bereich festgestellt haben, wurden nur in den wenigsten Effektivitätsstudien tatsächlich auch die Wahrnehmungen und Leistungen der Schüler/innen berücksichtigt. Liegen empirische Studien vor, dann zeigen sich zudem kontroverse Ergebnisse. Dies bestätigen auch die Resultate aus aktuellen Studien (Fend, 2004; Moser, Keller \& Tresch, 2002; Wössmann, 2005; Zahner Rossier et al., 2004). Dempster (2000, S. 56) kommt auf der Basis einer Metaanalyse von Studien in Australien, Neuseeland und England zum eher ernüchternden Schluss, dass die Effektivität von teilautonomen Schulen für die Schüler/innenleistungen kaum gegeben ist. Allerdings sei tendenziell von einer indirekten Funktionalität von Teilautonomie für die optimale Förderung der Kompetenzen der Schüler/innen auszugehen, indem es teilautonomen Schulen eher gelingt, eine optimale Lernumgebung herzustellen. Auch der von Kotthoff (2005) durchgeführte binationale Vergleich der beiden dezentralen Steuerungssysteme England und Schweden zeigt bilanzierend auf, dass die Zuweisung von Teilautonomie nicht direkt zu einer Optimierung der Qualität des Unterrichts und der Schüler/innenleistungen beiträgt, neben negativen Effekten (z.B. Verschärfung der funktionalen Differenzierung zwischen Schulleitung und Lehrpersonen, erhöhte Konkurrenz zwischen den Schulen mit gleichzeitig steigender Selektivität und Ungleichheit im Schulangebot) jedoch positive Wirkungen auf die Rahmenbedingungen der schulischen und unterrichtlichen Arbeit sichtbar werden (z.B. Intensivierung der Kooperation zwischen Lehrpersonen, professionalisierende Wirkung auf Schulleitungen). Die Ergebnisse der Studie von Ekholm (1997) weisen allerdings darauf hin, dass sich trotz neuem Steuerungssystem zwar Einstellungsveränderungen bei den Lehrpersonen und Schulleiterinnen und Schulleitern ergeben haben, die innere Arbeitsroutine bzw. die Arbeitskultur aber unverändert fortbesteht.

Zusammenfassend kann festgehalten werden, dass die empirische Befundlage zur Analyse der Effektivität von teilautonomen Schulen für die Schüler/innenleistungen und die Gestaltung einer förderlichen Lernumgebung nicht nur inkonsistent, sondern auch unzureichend ist. Neben den häufig zitierten Kritik- 
punkten wie ungenügende Berücksichtigung von multiplen Outputkriterien und Entwicklungsverläufen (Luyten, Visscher \& Witziers, 2005; Teddlie \& Reynolds, 2001) ist insbesondere virulent, dass der Implementierungsgrad der formalen Vorgaben der Schulen sowie die Umsetzung der Entscheidungs- und Handlungsspielräume in den Schulen häufig nicht differenziert genug untersucht worden sind und die Varianz innerhalb der jeweiligen Systeme nicht genügend Berücksichtigung gefunden hat. Dies ist in dem Sinne problematisch, als die Zuweisung von Teilautonomie einzig ein strukturelles Steuerungselement auf der Makroebene darstellt, die Rekontextualisierung dieser Vorgaben in der einzelnen Schule und ihren Arbeitsprozessen (Mesoebene) aber einen gegenüber den strukturellen Aspekten dominanten Einfluss auf die Lernergebnisse der Schüler/innen aufweist (Fend, 1986, 2006). In diesem Sinne ist die Analyse der Prozessstuktur und Funktionsweisen von Schulen in der Umsetzung der rechtlichen Vorgaben für die Beurteilung der Effektivität von Teilautonomie von zentraler Bedeutung. Zudem fehlen Studien, in denen die Effektivität der Umsetzung teilautonomer Vorgaben insbesondere aus der Perspektive der Schüler/innen untersucht worden sind.

In dieser Studie stehen die Gymnasien des Kantons Zürich im Fokus. Als teilautonome Schulen werden sie mit Erlass des Mittelschulgesetzes 1999 (Kanton Zürich, 1999/2000) selbstständig geleitet und haben den Auftrag zur Qualitätssicherung. Die Schulleitung ist für die pädagogische, administrative und finanzielle Führung der Schule verantwortlich und hat u.a. die Aufgaben, das Unterrichtsangebot festzulegen, Lehrpersonen mit befristeter Anstellung und das administrative/technische Personal zu ernennen oder zu entlassen. Zudem hat sie ein Antragsrecht auf Ernennung und Entlassung der Lehrpersonen mit unbefristeter Anstellung. Das Gesetz verpflichtet die Lehrpersonen, die ihre Mitwirkungsrechte im Gesamtkonvent und in Klassenkonventen ausüben, u.a. zusätzliche Funktionen und Aufgaben im Rahmen der Schulentwicklung zu übernehmen und mit der Schulleitung und dem Lehrerkollegium zusammenzuarbeiten.

Untersucht wird, a) inwiefern sich die Prozessstruktur der untersuchten Gymnasien - erfasst aus Sicht der Lehrpersonen - unterscheidet und b) inwiefern sich die Prozessstruktur der Gymnasien in den Wahrnehmungen der Schüler/innen zu Schule und Unterricht im Gymnasium abbildet. Damit wird systematisch die Varianz innerhalb eines formalen Regelsystems untersucht und die Varianz zwischen verschiedenen Regelsystemen, z.B. zwischen einem dezentralen und zentralen Regelsystem, vernachlässigt mit dem Ziel, die Heterogenität der Umsetzung teilautonomer Vorgaben und ihrer Effekte in Einzelschulen zu untersuchen. Mit der Fokussierung auf die Schul- und Unterrichtserfahrungen der Schüler/innen kommen wichtige Ergebniskriterien für die Beurteilung des Lernangebotes in das Blickfeld. 


\section{Prozessstruktur und Funktionsweisen von Schulen. Theoretische und empirische Befunde}

In der Literatur liegen einzelne theoretische Modelle vor, um die Prozessstruktur und Funktionsweisen von Schulen zu beschreiben und im Hinblick auf die Zielerreichung zu untersuchen. So haben Cheung und Cheng (2002) ein Konzept des «multilevel self-management in school» entwickelt, welches auf Qualitätdimensionen auf den drei Ebenen Schule, Team und Individuum basiert. Schulen mit hohem «multilevel self-management» sind jene, die auf allen drei Levels die höchsten Werte erzielt haben. Die Schulen mit dem geringsten Multilevel-SelfManagement sind die Schulen mit den niedrigsten Werten in allen drei Bereichen. Dazwischen können Schulen identifiziert werden, die in den einen Bereichen zwar hohe Werte, in anderen Bereichen aber niedrige Werte erzielt haben. Ein Extremgruppenvergleich zwischen den Schulen zeigt, dass Schüler/innen in Schulen mit einem ausgeprägten Self-Management auf allen Ebenen bessere Schulabschlüsse erreicht haben als Schulen mit einem nur gering ausgeprägten Self-Management. Allerdings zeigt die Studie auch, dass die Schüler/innen in Schulen, die nur in einzelnen Bereichen ein hohes Self-Management aufweisen, vergleichbar gute Leistungen erbracht haben wie die Schüler/innen in Schulen mit einem auf allen Level hohen Self-Management.

Pang (2003) fokussiert die Funktionsweisen der Schulen. Er formuliert ein "theoretical framework of binding forces in school organizations» und untersucht den Effekt der vier verschiedenen Kohäsionskräfte, «Bureaucratic Linkage», "Cultural Linkage», «Tight Coupling» und «Loose Coupling» auf die Wahrnehmungen von Lehrpersonen zu "Ordnung und Disziplin», «Zusammengehörigkeitsgefühl», "Arbeitszufriedenheit» und "Lehrerengagement». Die Ergebnisse zeigen, dass zu den stärksten Kohäsionskräften die "Cultural Linkage», «Tight Coupling» und «Loose Coupling» zu zählen sind, während dem «Bureaucratic linkage» zwar positive Effekte auf "Lehrerengagement», aber negative auf das Zusammengehörigkeitsgefühl und die Arbeitszufriedenheit der Lehrpersonen aufweist. Insgesamt zeigen die Resultate im positiven Bereich nicht eine eindeutige Rangfolge hinsichtlich ihrer Effektivität für Schulprozesse. Hingegen erweisen sich sehr bürokratisch-fragmentierte Strukturen als ungünstig.

Eine Differenzierung von Schulen auf der Basis unterschiedlicher Entscheidungsstrukturen in den Schulen wird von Kuper (2002) beschrieben. Mit Hilfe von Clusteranalysen lassen sich vier Typen von Schulen entlang der beiden Dimensionen "Kollegiale Abstimmung» und "Individuelle Verantwortung» unterscheiden. Die «integrierten Schulen» weisen in beiden Bereichen die höchsten Werte auf, während dem die «schwachen Schulen» in beiden Bereichen die niedrigsten Werte erreichen. Im Gegensatz zu den beiden Schultypen «kleine Schulen» und "grosse Schulen» ergeben sich bei den beiden ersten Schultypen keine Unterschiede in der Schulgrösse. In den "grossen Schulen» hingegen wird die kollegiale Abstimmung als überdurchschnittlich, die individuelle Verantwortung 
als unterdurchschnittlich ausgeprägt wahrgenommen, wohingegen in den «kleinen Schulen» die individuelle Verantwortung als überdurchschnittlich, die kollegiale Abstimmung als unterdurchschnittlich eingeschätzt werden. Überdurchschnittlich ausgeprägt ist in den «integrierten Schulen» zudem eine in die internen Entscheidungen involvierte Leitung, wohingegen in den anderen drei Schultypen diesbezüglich kaum grosse Unterschiede bestehen. Die vier verschiedenen Schultypen wurden von Kuper in einem zweiten Schritt in Beziehung gesetzt zum Ausmass an Austausch über den Unterricht, wie ihn die Lehrpersonen in diesen Schulen wahrnehmen. Die Resultate zeigen, dass aus Sicht der Lehrpersonen in den integrierten Schulen der intensivste kollegiale Austausch über den Unterricht stattfindet, gefolgt von den «kleinen Schulen». In den Clustern der «grossen» und der «schwach integrierten Schulen» nimmt der Austausch zwischen den Lehrpersonen hingegen das geringste Ausmass an.

Studienübergreifend kann gesagt werden, dass die Schulen sich in ihrer Prozessstruktur und ihren Funktionsweisen systematisch voneinander unterscheiden. Untersucht wurden allerdings insbesondere die Effekte dieser Funktionsweisen in Bezug auf einzelne Aspekte des Handelns von Lehrpersonen, weniger hingegen hinsichtlich der Kompetenzen der Schüler/innen und ihrer Wahrnehmungen zu Schule und Unterricht. Als übergeordnetes Strukturmuster kann festgehalten werden, dass sich insbesondere die Schulen mit einem hohen Mass an dysfunktionalen oder funktionalen Prozessstrukturen identifizieren lassen, d.h. Schulen mit einem hohen Mass an bürokratischen Strukturen bei Pang (2003) oder Schulen, in denen das Multilevel-Self-Management niedrig ausgeprägt ist, wie bei Cheung und Cheng (2002). Bei Kuper (2002) ist es vor allem die «Integrative Schule», die die besten Resultate erzielt hat. Im Gegensatz zu diesen Schulen können die anderen Schulen weniger gut voneinander unterschieden werden.

Aufgrund dieser Ergebnisse kann für die hier präsentierte Studie davon ausgegangen werden, dass sich die untersuchten Gymnasien im Ausmass ihrer Implementation ausgewählter Prozessmerkmale von Teilautonomie systematisch unterscheiden. Zudem ist anzunehmen, dass sich diese Unterschiede der Implementation insbesondere in den Extremgruppen abbilden. Schüler/innen in Gymnasien, in denen zentrale Prozessmerkmale von Teilautonomie in stärkerem Maße implementiert sind, dürften einen günstigeren Lernkontext vorfinden als Schüler/innen in Gymnasien, in denen dies weniger der Fall ist.

\section{Forschungsinstrumentarium und Stichprobe}

Die Bearbeitung der hier interessierenden Fragestellungen erfolgt im Rahmen einer Längsschnittstudie im Kanton Zürich (2001-2004), in der u.a. die Schulund Unterrichtserfahrungen aus Sicht der Lehrpersonen und Schüler/innen systematisch erfasst worden sind (Maag Merki, 2006). Von den insgesamt 22 
Gymnasien (Vollerhebung im Kanton Zürich) können für die vorliegenden Analysen acht Gymnasien berücksichtigt werden (36.4\%). Diese acht Schulen haben sich, ergänzend zu der von der Bildungsdirektion des Kantons Zürich vorgegebenen Schüler/innenbefragung, freiwillig an der Lehrpersonenbefragung beteiligt. Sie bilden die Grundgesamtheit aller zürcherischen Gymnasien hinsichtlich verschiedener Merkmale (z.B. Zusammensetzung der Schüler/innen, angebotene Maturitätsprofile, Geschlecht der Lehrpersonen, Pensenverteilung der Lehrpersonen) gut ab, so dass trotz eines möglichen Selbstselektionseffektes von einer guten Datengrundlage ausgegangen werden kann (Halbheer \& Kunz, 2004).

In jedem Gymnasium wurden alle Lehrpersonen in die Befragung einbezogen. Der Rücklauf der Fragebogen kann mit 60.6\% als gut bezeichnet werden. In keinem der Gymnasien wurde zudem die Schwelle von 50\% Rücklauf unterschritten. Die Stichprobe umfasst 409 Lehrpersonen (54.7\% männliche, 45.3\% weibliche Lehrpersonen). Nur ein Viertel der Lehrpersonen ist weniger als fünf Jahre an den untersuchten Schulen beschäftigt. Der Grossteil der befragten Lehrpersonen unterrichtet damit schon seit langer Zeit an den Gymnasien und verfügt über einen breiten Erfahrungshintergrund zur Beurteilung der schulischen Praktiken in den einzelnen Gymnasien.

An der Schüler/innenbefragung nahmen alle Schüler/innen aller Abschlussklassen der untersuchten Gymnasien teil. Da die Datenerhebung während einer offiziellen Klassenstunde stattgefunden hat, ist der durchschnittliche Rücklauf von über 90\% der Schüler/innen sehr hoch. Analysiert werden die Daten jener Schüler/innen, von denen Daten aus beiden Erhebungszeitpunkten vorliegen. Für die hier interessierenden acht Gymnasien sind das 365 Schüler/innen (65.3\% weiblich, 34.7\% männlich). Nicht nur hinsichtlich der Verteilung der Geschlechter, auch hinsichtlich des Notendurchschnittes im letzten Zeugnis oder des familiären Bildungshintergrundes weist die hier interessierende Schüler/innenstichprobe vergleichbare Parameter wie die Untersuchungsgruppe aller Gymnasien auf.

Als Indikatoren für die Beurteilung des Lernkontextes aus Sicht der Schüler/innen wurden relevante Dimensionen zur Erfassung der Unterrichtsqualität (Clausen, 2002; Ditton, 2000; Gruehn, 2000; Helmke, 2003) und des Schulund Klassenklimas (Eder, 1996; Fend \& Prester, 1986) aus der Perspektive der Schüler/innen berücksichtigt. Die entsprechenden Skalen und Beispielitems sind in Tabelle 1 zusammengestellt. Sie wurden zum zweiten Erhebungstermin im Frühjahr 2004 kurz vor den Maturitätsprüfungen der Schüler/innen während einer offiziellen Schulstunde erfasst. 
Tabelle 1: Indikatoren zur Erfassung von Schul-und Unterrichtsklima, Unterrichtsgestaltung und Zufriedenheit und Woblbefinden; Beispielitem; Anzahl Probandlinnen (N), Mittelwert (M), Standardabweichung (SD), F-Wert; Anzahl Items, Reliabilität ( $R$; Cronbachs Alpha)

\begin{tabular}{|c|c|c|c|c|c|c|c|}
\hline $\begin{array}{l}\text { Dimen- } \\
\text { sion }\end{array}$ & Beispielitem & $\mathrm{N}$ & $\mathrm{M}$ & SD & $\begin{array}{c}F(p)^{1} \\
d f=7\end{array}$ & $\begin{array}{l}\text { An- } \\
\text { zahl } \\
\text { Items }\end{array}$ & $\mathrm{R}$ \\
\hline $\begin{array}{l}\text { Anony- } \\
\text { mität }\end{array}$ & $\begin{array}{l}\text { An dieser Schule muss sich je- } \\
\text { der/jede allein durchkämpfen, } \\
\text { auf Unterstützung kann er/sie } \\
\text { wenig rechnen.* } \\
\text { Basis: (Fend, 1977) }\end{array}$ & 386 & 2.11 & .56 & $\begin{array}{c}8.39 \\
(.000)\end{array}$ & 4 & .70 \\
\hline $\begin{array}{l}\text { Anre- } \\
\text { gung }\end{array}$ & $\begin{array}{l}\text { Kulturelle Aktivitäten haben an } \\
\text { unserer Schule einen hohen Stel- } \\
\text { lenwert.* } \\
\text { Basis: (Eder, 1998) }\end{array}$ & 387 & 2.42 & .47 & $\begin{array}{l}14.66 \\
(.000)\end{array}$ & 4 & .71 \\
\hline $\begin{array}{l}\text { Mit- } \\
\text { sprache }\end{array}$ & $\begin{array}{l}\text { An unserer Schule haben die } \\
\text { Schüler/innen bzw. ihre Vertre- } \\
\text { ter/innen der SO viel Einfluss.* } \\
\text { Basis: (Eder, 1998; Fend, 1977) }\end{array}$ & 385 & 2.43 & .76 & $\begin{array}{l}16.34 \\
(.000)\end{array}$ & 4 & .79 \\
\hline Wärme & $\begin{array}{l}\text { Die Stimmung an unserer Schule } \\
\text { ist meistens ( } 1=\text { heiter, fröhlich, } \\
\ldots 5 \text { =gedrückt, lustlos) } \\
\text { Basis: (Eder, 1998) }\end{array}$ & 388 & 3.57 & 0.61 & $\begin{array}{c}6.99 \\
(.000)\end{array}$ & 9 & .88 \\
\hline $\begin{array}{l}\text { S-S-Be- } \\
\text { ziehung }\end{array}$ & $\begin{array}{l}\text { Freundschaften zwischen den } \\
\text { Schülerinnen und Schülern sind } \\
\text { bei uns nur oberflächlich, letzten } \\
\text { Endes arbeitet jeder gegen jeden. } \\
(-)^{* *} \\
\text { Basis: Eigenentwicklung }\end{array}$ & 388 & 3.77 & 0.68 & $\begin{array}{c}7.68 \\
(.000)\end{array}$ & 6 & .78 \\
\hline $\begin{array}{l}\text { LP-S-Be- } \\
\text { ziehung }\end{array}$ & $\begin{array}{l}\text { Lehrer und Lehrerinnen achten } \\
\text { unsere Meinungen.** } \\
\text { Basis: Eigenentwicklung }\end{array}$ & 388 & 3.44 & 0.57 & $\begin{array}{l}2.06 \\
(.000)\end{array}$ & 12 & .86 \\
\hline $\begin{array}{l}\text { Arbeits- } \\
\text { reflexion }\end{array}$ & $\begin{array}{l}\text { Im Unterricht blicken wir immer } \\
\text { wieder auf unsere Lernmethoden } \\
\text { und Lerntätigkeiten zurück.*** } \\
\text { Basis: Eigenentwicklung }\end{array}$ & 388 & 1.31 & 0.42 & $\begin{array}{c}2.14 \\
(.039)\end{array}$ & 5 & .75 \\
\hline $\begin{array}{l}\text { Elabora- } \\
\text { tion }\end{array}$ & $\begin{array}{l}\text { Wir werden oft aufgefordert, } \\
\text { Fachkenntnisse bei aktuellen } \\
\text { Ereignissen und alltäglichen Si- } \\
\text { tuationen anzuwenden. }{ }^{* * *} \\
\text { Basis: Eigenentwicklung }\end{array}$ & 388 & 2.35 & 0.45 & $\begin{array}{c}3.92 \\
(.000)\end{array}$ & 7 & .73 \\
\hline $\begin{array}{l}\text { Gene- } \\
\text { tisch-So- } \\
\text { kra- } \\
\text { tisches } \\
\text { Vorge- } \\
\text { hen }\end{array}$ & $\begin{array}{l}\text { Im Unterricht lassen uns unsere } \\
\text { Lehrpersonen auch einmal mit } \\
\text { unseren eigenen Vermutungen in } \\
\text { die Irre gehen, bis wir es selbst } \\
\text { merken.*** } \\
\text { Basis: (Clausen, 2002) }\end{array}$ & 388 & 2.14 & 0.52 & $\begin{array}{c}4.52 \\
(.000)\end{array}$ & 4 & .68 \\
\hline
\end{tabular}




\begin{tabular}{|l|l|c|c|c|c|c|c|}
\hline $\begin{array}{l}\text { Dimen- } \\
\text { sion }\end{array}$ & Beispielitem & M & SD & $\begin{array}{c}\mathbf{F}(\mathbf{p})^{1} \\
\mathbf{d f}=7\end{array}$ & $\begin{array}{c}\text { An- } \\
\text { zahl } \\
\text { Items }\end{array}$ & $\mathbf{R}$ \\
\hline $\begin{array}{l}\text { Selbstak- } \\
\text { tivität }\end{array}$ & $\begin{array}{l}\text { Im Unterricht gibt es für uns } \\
\text { Schülerinnen und Schüler im- } \\
\text { mer wieder Gelegenheiten, ei- } \\
\text { gene Ideen zu realisieren..*** } \\
\text { Basis: Eigenentwicklung }\end{array}$ & 388 & 2.24 & 0.47 & $\begin{array}{c}7.70 \\
(.000)\end{array}$ & 5 & .71 \\
\hline $\begin{array}{l}\text { Self-mo- } \\
\text { nitoring }\end{array}$ & $\begin{array}{l}\text { Wir Schülerinnen und Schüler } \\
\text { beurteilen unsere Arbeiten im- } \\
\text { mer wieder gegenseitig.*** } \\
\text { Basis: Eigenentwicklung }\end{array}$ & 388 & 1.77 & 0.52 & $\begin{array}{c}5.77 \\
(.000)\end{array}$ & 4 & .68 \\
\hline $\begin{array}{l}\text { Förder- } \\
\text { haltung }\end{array}$ & $\begin{array}{l}\text { Bei unseren Lehrpersonen zäh- } \\
\text { len nur die Spitzenschülerinnen } \\
\text { und -schüler. (-)** } \\
\text { Basis: (Fend, 1977) }\end{array}$ & 388 & 3.24 & .70 & $\begin{array}{c}5.22 \\
(.000)\end{array}$ & 5 & .80 \\
\hline $\begin{array}{l}\text { Motivie- } \\
\text { rungsfä- } \\
\text { higkeit }\end{array}$ & $\begin{array}{l}\text { Unsere Lehrpersonen können } \\
\text { Schülerinnen und Schüler } \\
\text { manchmal richtig begeistern.** } \\
\text { Basis: (Baumert et al. 1997; } \\
\text { Prenzel et. al. 1996) }\end{array}$ & 388 & 2.20 & 0.50 & $\begin{array}{c}1.92 \\
(.066)\end{array}$ & 8 & .86 \\
\hline $\begin{array}{l}\text { Autono- } \\
\text { mieun- } \\
\text { terstüt- } \\
\text { zung }\end{array}$ & $\begin{array}{l}\text { Im Unterricht habe ich oft Ge- } \\
\text { legenheit, mich mit interessan- } \\
\text { ten Aufgaben oder Inhalten ein- } \\
\text { gehender zu beschäftigen.** } \\
\text { Basis: (Prenzel et al., 1996) }\end{array}$ & 388 & 2.22 & 0.50 & $\begin{array}{c}6.95 \\
(.000)\end{array}$ & 4 & .66 \\
\hline
\end{tabular}

Antwortskalen:

${ }^{*} 1=$ trifft gar nicht zu, $\ldots 4=$ trifft genau zu; ${ }^{* *} 1=$ nie, $\ldots 5=$ sehr oft; ${ }^{* * *}$ Dies stimmt bei. $\ldots 1=$ keinen oder sehr wenigen meiner Lehrpersonen, ... $4=$ den allermeisten oder allen meiner Lehrpersonen

1 Varianzanalyse: Unterschiede zwischen Schulen ohne Kontrolle Hintergrundvariablen

Zur Erfassung des Implementierungsgrades von Teilautonomie in der einzelnen Schule wurde das Ausmass an Kooperation zwischen den Lehrpersonen in verschiedenen Bereichen untersucht. Die Kooperation von Lehrpersonen mit der Schulleitung und dem Lehrerkollegium ist seit dem Erlass des Mittelschulgesetz 1999 verpflichtende Aufgabe der Lehrpersonen (Kanton Zürich, 1999/2000). Kooperation in den einzelnen Schulen kann zudem als wichtiger, wenn auch nicht einziger Aspekt teilautonomer Schulen interpretiert werden (Dempster, 2000; Seashore \& Leithwood, 1998; Silins, Zarins \& Mulford, 2002).

Auf Seiten der Lehrpersonen wurde das Instrumentarium zur Erfassung der Lehrerkooperation und das Verfahren zur Ermittlung von Kooperationslevels der einzelnen Schulen von Steinert et al. (2006) einbezogen, welches es erlaubt, das Ausmass an Kooperation zwischen Lehrpersonen in verschiedenen Bereichen innerhalb einer Schule zu bestimmen. Dieses Instrumentarium beinhaltet 20 Items zu Aspekten der Kooperation zu Unterricht, Organisation, Schulleitungshandeln und Qualitätssicherung, die auf individueller Ebene erfasst und auf 
Schulebene aggregiert worden sind. Dabei wird das Ausmass der Zustimmung zu den Kooperationsaufgaben als Indikator für die Lehrerkooperation im Kollegium genutzt. Wenn 50\% und mehr der Lehrpersonen eines Kollegiums ein Kooperationsitem mit "trifft eher zu» und "trifft genau zu» beanworten, wird dies dahingehend bewertet, dass das Kollegium in dieser Frage kooperiert, anderenfalls nicht. Die Skalierung der Kooperationsitems und die Zuweisung der einzelnen Schulen zu den Kooperationsniveaus wurden analog zur Kompetenzmessung in der Schulleistungsforschung auf der Basis der Item-Response-Theorie durchgeführt. So wurden aufgrund der geschätzten Itemschwierigkeiten der Kooperationsitems diese in eine Rangfolge gebracht, wobei die Items mit ähnlicher Schwierigkeit zu homogenen Itemgruppen zusammengefasst worden sind. Hieraus resultierten die Niveaustufen der Lehrerkooperation (siehe Tabelle 2 in Steinert et al., 2006, S. 194).

Insgesamt werden fünf verschiedene Niveaustufen unterschieden: Die unterste Stufe der Lehrerkooperation ist ausschließlich negativ definiert und wird als "Fragmentierung» bezeichnet. Schulen auf dieser untersten Stufe zeichnen sich aus durch das Fehlen eines gemeinsamen Schulprofils. In der fachspezifischen Arbeit dominiert isoliertes Lehrerhandeln und die Zusammenarbeit findet nur punktuell statt. Schulen auf dem ersten Kooperationslevel "Differenzierung» haben ein globales Zielkonzept, formal geregelte Arbeitsabläufe und Kommunikationsformen. Die Lehrerkooperation vollzieht sich aber hauptsächlich innerhalb der Fach- und Jahrgangsgrenzen. Schulen auf der zweiten Niveaustufe der Lehrerkopperation "Koordination» erfüllen die Anforderungen auf der ersten Stufe. Darüber hinaus weisen sie eine umfassende Information über Arbeitsabläufe und Arbeitsergebnisse auf. Zudem lassen sich erste Aspekte zu einer Koordination von Ressourcen und zu Teamarbeit identifizieren. Auf dem dritten Kooperationslevel "Interaktion» finden sich Kooperationen zu Unterricht innerhalb der einzelnen Fächer und Jahrgänge wie auch fach- und jahrgangsübergreifend. Weiter werden Selbst- und Fremdevaluationen durchgeführt, die für Personal- und Unterrichtsentwicklung genutzt werden. Es lassen sich zudem Ansätze zu einer kollegialen Handlungsregulation und Verbesserung individuellen Lehrerhandelns identifizieren. Schulen auf dem höchsten Kooperationslevel "Integration» zeichnen sich durch eine systematische bereichsspezifische und bereichsübergreifende Kooperation unter den Lehrpersonen in verschiedenen Themenbereichen aus. Gegenseitige Hospitationen sind ein selbstverständlicher Teil der Arbeit, zudem wird das Kollegium als soziale Ressource genutzt, indem gemeinsame Strategien zur Bewältigung beruflicher Probleme erarbeitet werden.

Die Kooperationsfähigkeiten der einzelnen Schulen werden aus den Antworten des Kollegiums auf alle Kooperationsitems geschätzt. Die Zuordnung einer Schule zu einer Kooperationsstufe erfolgt auf der Basis der Itemschwierigkeiten. «Eine Schule wird dann einer Kooperationsstufe zugewiesen, wenn das geschätzte Kooperationsniveau der Schule über der unteren Schwelle der Niveaustufen liegt. Dies bedeutet, dass eine Schule dann einer Kooperationsstufe zu- 
geordnet wird, wenn das Kollegium wenigstens das einfachste Item der jeweiligen Stufe mit einer Wahrscheinlichkeit von 50\% erfüllt» (Steinert et al., 2006, S. 193). Innerhalb der so definierten Niveaustufen kann eine Schule mit der angegebenen Wahrscheinlichkeit nur einen Teil oder auch alle Kooperationsanforderungen erfüllen. Da die geschätzten Itemschwierigkeiten und Kooperationsniveaus der Schulen auf derselben Skala abgebildet werden, können die in Abbildung 1 jeweils angeführten Kooperationsniveaus der Schulen durch die Itemschwierigkeiten qualifiziert und den entsprechenden Abschnitten auf der Skala zugeordnet werden (Steinert et al., 2006, S. 194). Die jeweils typischen Items und die Zuordnung der acht Gymnasien zu den einzelnen Levels sind in Abbildung 1 dargestellt: Eine Schule erreichte den Level 1 «Differenzierung», zwei Schulen den Level 2 «Koordination», vier Schulen den Level 3 «Interaktion» und eine Schule den höchsten Level 4 «Integration». Keine Schule erreichte einzig die Niveaustufe 0 «Fragmentierung».

\section{Auswertungsstrategien}

Für die Analyse der Differenzen zwischen den Schulen zur Bewertung der Schulund Unterrichtsqualität wurden auf individueller Ebene der Schüler/innendaten Multiple Regressionsanalysen gerechnet. Zur Vergleichbarkeit der Schulen wurden verschiedene Hintergrund-Variablen als unabhängige Variablen berücksichtigt: Geschlecht und familiärer Bildungshintergrund der Schüler/innen, Profil des gewählten gymnasialen Abschlusses und fünf Indikatoren zur Erfassung der Freizeitaktivitäten der Jugendlichen (z.B. Mitarbeit in einem Verein) (Maag Merki, 2006, S. 56). Dabei wurden die standardisierten Residuen abgespeichert. Die Regressionsresiduen kennzeichnen die Abweichung eines empirischen Wertes vom vorgesagten Wert. Sie enthalten damit, neben Messfehlern der Kriteriumsvariable, vor allem Anteile der Kriteriumsvariable, die durch die Prädiktorvariablen nicht erfasst worden sind, sondern durch andere, mit den Prädiktorvariablen nicht zusammenhängenden Merkmale erklärt werden können (Bortz, 1999, S. 199). 
Niveaustufen der Lehrerkooperation. Beispielitems für die Kooperationsanforderungen und Kooperationsniveaus der Schulen*

Integration:

Gegenseitige Unterrichtsbesuche sind ein selbstverständlicher

Teil unserer Arbeit.

Wir erarbeiten gemeinsame Strategien zur Bewältigung beruflicher Schwierigkeiten

\section{Interaktion:}

Die Zusammenarbeit im Kollegium orientiert sich fast ausschließlich an den Fächern (umgepolt).

Wir haben eine fachübergreifende Zusammenarbeit, die sich an gemeinsamen Themen orientiert.

\section{Koordination:}

Im Kollegium gibt es Gruppen, die nur wenig miteinander zu tun haben wollen (umgepolt).

Ergebnisse aus Arbeitsgruppen werden regelmäßig im Kollegium bekannt gegeben.

\section{Differenzierung:}

Bei der Festlegung des Stundenplans werden wir ausreichend beteiligt.

Wir haben eine gute fachspezifische Zusammenarbeit.

\section{Fragmentierung:}

Die Anforderung keines Items wird erfüllt

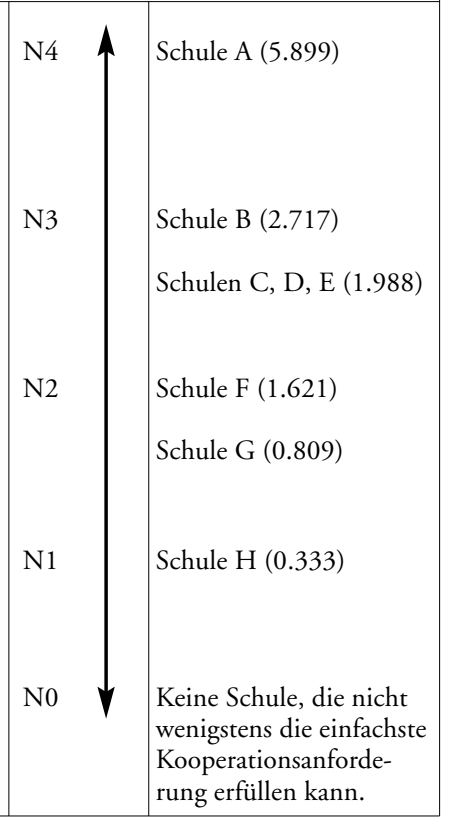

Abbildung 1: Zuordnung der Schulen zu den Niveaustufen der Lehrerkooperation. Beispielitems und die geschätzten Logits für die jeweiligen Kooperationsniveaus in Klammern. Antwortkategorien: 1=trifft gar nicht zu ... 4=trifft genau zu

Die standardisierten Residuen wurden in einem zweiten Schritt auf Schulebene aggregiert. Damit erhält man die um den Einfluss der Hintergrundvariablen bereinigten Werte der einzelnen Schulen bzw. den Erwartungswert der einzelnen Schule. Ein Schulvergleich ist damit fair, da allfällige unterschiedliche $\mathrm{Zu}-$ sammensetzungen in der Schülerschaft der Schulen rechnerisch neutralisiert worden sind. Die Qualität dieses Vorgehens ist abhängig von der Relevanz der berücksichtigten Hintergrundvariablen. Die in dieser Studie kontrollierten Faktoren haben sich in Bezug auf die Wahrnehmung von Schule und Unterricht als bedeutsam erwiesen (Maag Merki, 2006). Werte im positiven Bereich deuten darauf hin, dass diese Schulen in der entsprechenden Dimension den Erwartungswert übertreffen, Schulen im negativen Bereich sind Schulen, deren Ergebnisse unterhalb des Erwartungswertes liegen. Dieses Vorgehen wird regelmässig in nationalen und internationalen Leistungsvergleichsstudien wie beispielsweise PISA zur Rückmeldung der schulspezifischen Ergebnisse an die einzelne Schule angewandt (Watermann \& Stanat, 2004).

Aufgrund dessen, dass in dieser Studie einzig acht Gymnasien untersucht werden konnten und aus diesem Grund auf einzelnen Niveaustufen teilweise nur 
eine Schule zugeordnet ist, lassen die nachfolgenden Analysen lediglich explorative Aussagen und die Formulierung von Hypothesen zu, die wiederum an einer grösseren Stichprobe zu untersuchen sind.

\section{Ergebnisse}

Die Profile der einzelnen Schulen sind in den nachfolgenden drei Abbildungen sichtbar (vgl. Abbildung 2, 3, 4). In allen untersuchten Dimensionen zeichnen sich systematische Unterschiede zwischen den Schulen ab (vgl. Tabelle 1). in Kapitel 2).

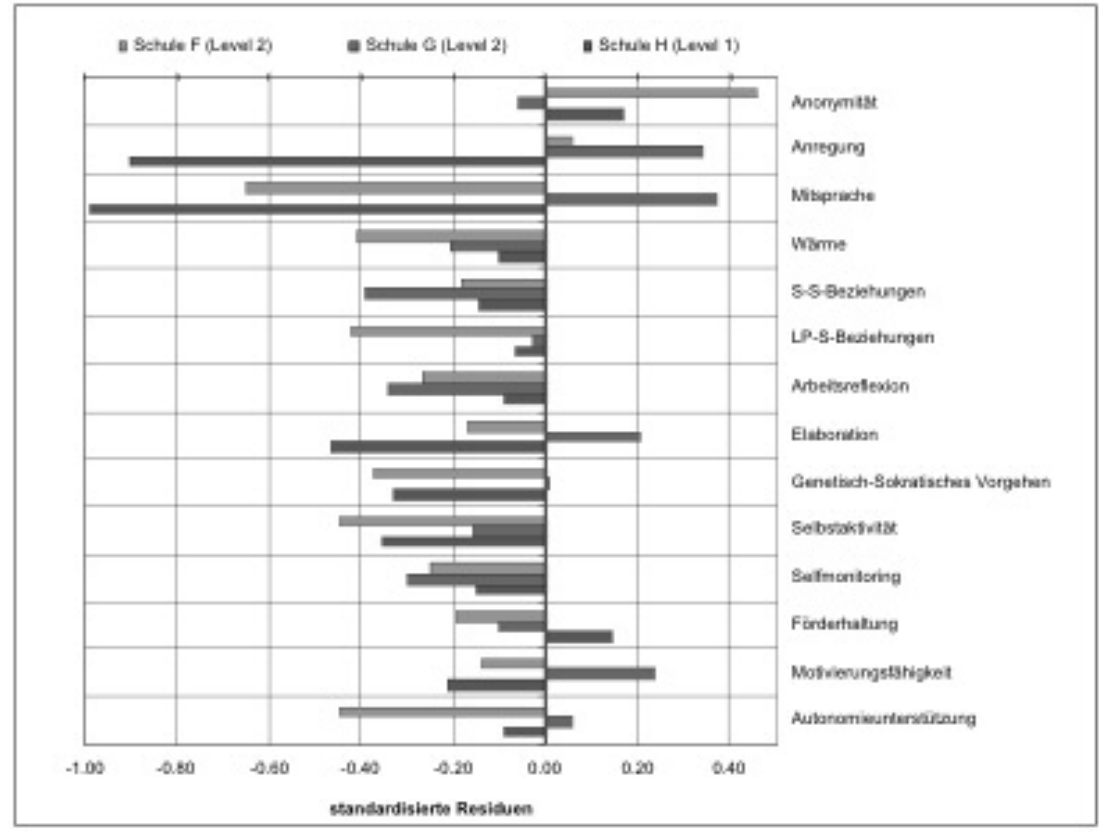

Abbildung 2: Profile der Level-1-Schule und der Level-2-Schulen

Werden die Profile der Level-1- und 2-Schulen betrachtet (vgl. Abbildung 2), so fällt auf, dass die Schulen fast durchgehend negativere Beurteilungen als der Durchschnitt aller Schulen aufweisen und - gemessen an ihren Hintergrundmerkmalen - in den meisten Dimensionen erwartungswidrig niedrige Ergebnisse erzielen. In einzelnen Fällen sind die Effekte sehr gross. So beurteilen die Schüler/innen der Level-1-Schule H ihre Mitsprachemöglichkeiten um fast eine Standardabweichung geringer ein als der Durchschnitt aller Schulen. Ein vergleichbares Ergebnis zeigt sich bei der Einschätzung des Anregungsgehalts in der Schule. Die Schüler/innen in dieser Schule geben zudem systematisch weniger häufig an, 
dass sie in ihrem Unterricht verschiedene Fachinhalte miteinander verknüpfen («Elaboration»-0.47), eigene Lernaktivitäten realisieren («Selbstaktivität» -0.36) und bei ihren Lehrpersonen erleben, dass sie in der Erarbeitung eines Lernstoffes auch einmal «in die Irre gehen können», bis sie es selber merken ("Genetisch-sokratischer Vorgehen» -0.33). Weiter nehmen sie bei ihren Lehrpersonen systematisch weniger häufig wahr, dass diese Freude am Unterrichtsstoff haben und ihre Schüler/innen begeistern können («Motivierungsfähigkeit»-0.21).

Bei den Ergebnissen der Level-2-Schule F fällt der hohe Wert in der Einschätzung der Anonymität und in den wahrgenommenen Mitsprachemöglichkeiten in der Schule auf. Die Schüler/innen geben damit weitaus häufiger als alle Schüler/innen an, dass sie sich in ihrer Schule alleine gelassen fühlen ("Anonymität» 0.45) und weniger Möglichkeiten zur Mitbestimmung haben («Mitsprache»-0.65). Weitere substanzielle Abweichungen im negativen Bereich sind bei dieser Schule zudem bei verschiedenen weiteren Indikatoren zu finden, so bei «Wärme» (-0.41), «LP-S-Beziehungen» (-0.42), «Arbeitsreflexion» (-0.27), «Genetisch-sokratisches Vorgehen» (-0.38), «Selbstaktivität» (-0.45), "Selfmonitoring» (-0.25), «Autonomieunterstützung» (-0.45).

Die Ergebnisse der Level-2-Schule G liegen zwar ebenfalls mehrheitlich im negativen Bereich, aber einzelne substanzielle Abweichungen im positiven Bereich sind vorzufinden. So erleben die Schüler/innen überdurchschnittlich häufig eine angeregte Schulkultur («Anregung» 0.34) und positive Mitsprachemöglichkeiten (0.37) sowie eine ausgesprochene Motivierungsfähigkeit bei ihren Lehrpersonen (0.24). Sie nehmen häufiger als der Durchschnitt aller Schüler/innen wahr, dass sie in ihrem Unterricht Fachinhalte aus unterschiedlichen Fächern miteinander oder mit Erfahrungen aus dem Alltag verknüpfen («Elaboration» $(0.20)$.

Zusammenfassend zeigt sich, dass Schüler/innen in Schulen mit eher niedrigem Kooperationslevel fast durchgehend ein deutlich negativeres Schul- und Klassenklima wie auch eine deutlich geringer ausgeprägte selbstaktivierende und unterstützende Unterrichtsgestaltung wahrnehmen als der Durchschnitt aller Schüler/innen in den untersuchten Gymnasien. Die Schulen auf Level 2 weisen teilweise sogar grössere Negativabweichungen auf als die Level-1-Schule H.

Werden die Profile der Level-3-Schulen betrachtet (vgl. Abbildung 3), dann zeigt sich, dass das Ergebnismuster der vier Schulen, die dieser Niveaustufe zugeteilten sind, unterschiedlich ausfällt. Durchgehend im überdurchschnittlichen oder durchschnittlichen Bereich befinden sich die Schulen D und E. Die Schüler/innen in diesen Schulen nehmen tendenziell durchschnittlich bis überdurchschnittlich positive Beurteilungen der Schul- und Unterrichtsgestaltung vor, wobei die positiven Abweichungen der Schule D tendenziell grösser sind als diejenigen der Schule E. Auffallend sind insbesondere die Werte der Schule D in den Bereichen «Mitsprache» (0.47), «Wärme» (0.25), «LP-S-Beziehungen» (0.42), «Elaboration» (0.22), «Genetisch-Sokratisches Vorgehen» (0.34), «Selbstaktivität» (0.23), «Selfmonitoring» (0.33) und «Förderhaltung» (0.22). Bei der 
Schule E sind insbesondere die Werte bei «Anregung» (0.45), «S-S-Beziehungen» (0.39), "Arbeitsreflexion» (0.35) und "Autonomieunterstützung» (0.22) auffallend.

Im Gegensatz zu den Schulen D und E weisen die Schulen B und C fast durchgehend erwartungswidrig niedrigeWerte auf. Bei der Schule B sind die negativen Werte inbesondere in den Bereichen "Anregung» (-0.59), "Mitsprache» (-0.36), «S-S-Beziehungen» (-0.29), «LP-S-Beziehungen» (-0.29), «Elaboration» $(-0.29)$, «Genetisch-sokratisches Vorgehen» (-0.29) und "Selfmonitoring» $(-0.24)$ bemerkenswert. Die Werte der Schule $\mathrm{C}$ wiederum sind bis auf die Bereiche «Mitsprache» $(0.35)$, «Wärme» $(0.08)$ in allen untersuchten Dimensionen deutlich im negativen Bereich (Werte zwischen -0.21 und -0.72).

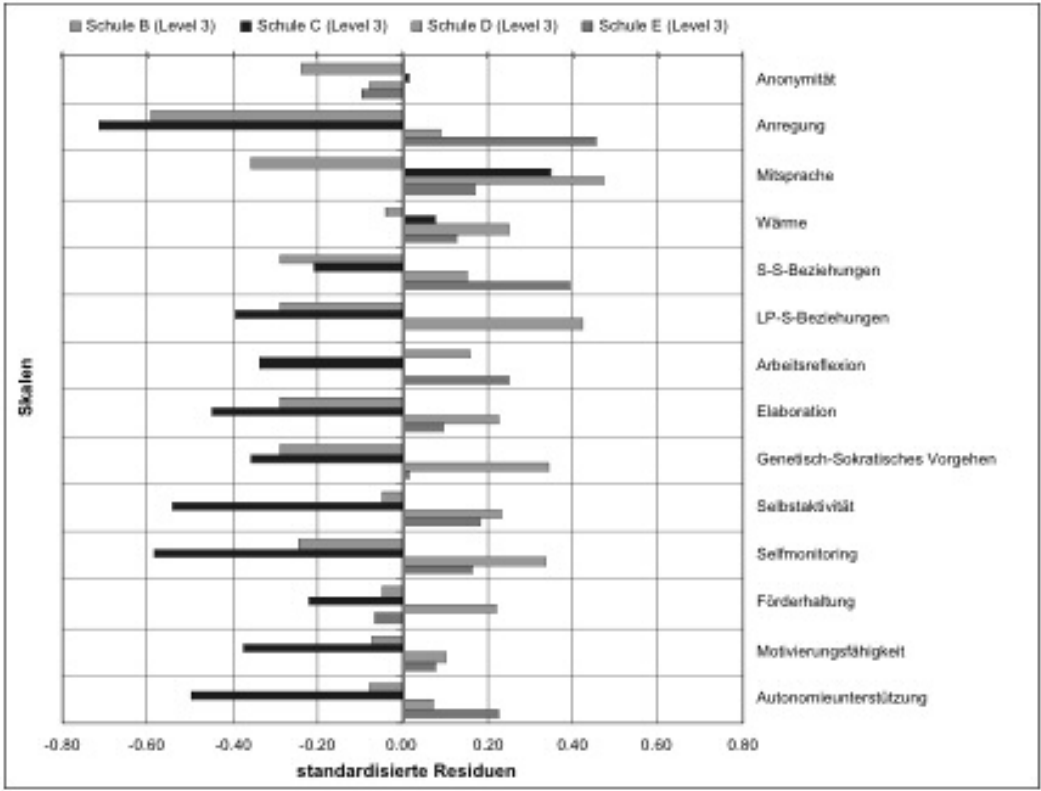

\section{Abbildung 3: Profile der Level-3-Schulen}

In Abbildung 4, in der die einzige Schule auf Kooperationslevel 4 darstellt ist, zeigt sich ein klares und in den meisten Fällen erwartungskonformes Bild. Die Level-4-Schule A weist im Vergleich zu allen untersuchten Schulen praktisch durchgehend erwartungskonforme überdurchschnittliche Ergebnisse auf. Die Schüler/innen dieser Schule nehmen somit deutlich häufiger einen unterstützenden, selbstaktivierenden Unterricht und ein positives Schul- und Klassenklima wahr als die Schüler/innen aller untersuchten Schulen (Werte zwischen 0.22 und 0.63). Die Effekte sind bis auf wenige Ausnahmen («Anregung» 0.14, «Arbeitsreflexion» 0.07) bedeutsam. Abweichend von dieser Ergebnisstruktur 
sind die Ergebnisse bei der Beurteilung der Beziehungen zwischen den Schüler/innen (-0.47) und ihrer Mitsprachemöglichkeiten in der Schule (-0.33), die deutlich unterdurchschnittlich ausfallen. In einzelnen Dimensionen weisen die Level-3-Schulen D und E (vgl. Abbildung 3) zwar grössere Positivabweichungen als die Level 4-Schule A auf. Über alle Dimensionen betrachtet erreicht die Schule A aber am häufigsten (in acht Dimensionen) die positivsten Werte aller Schulen.

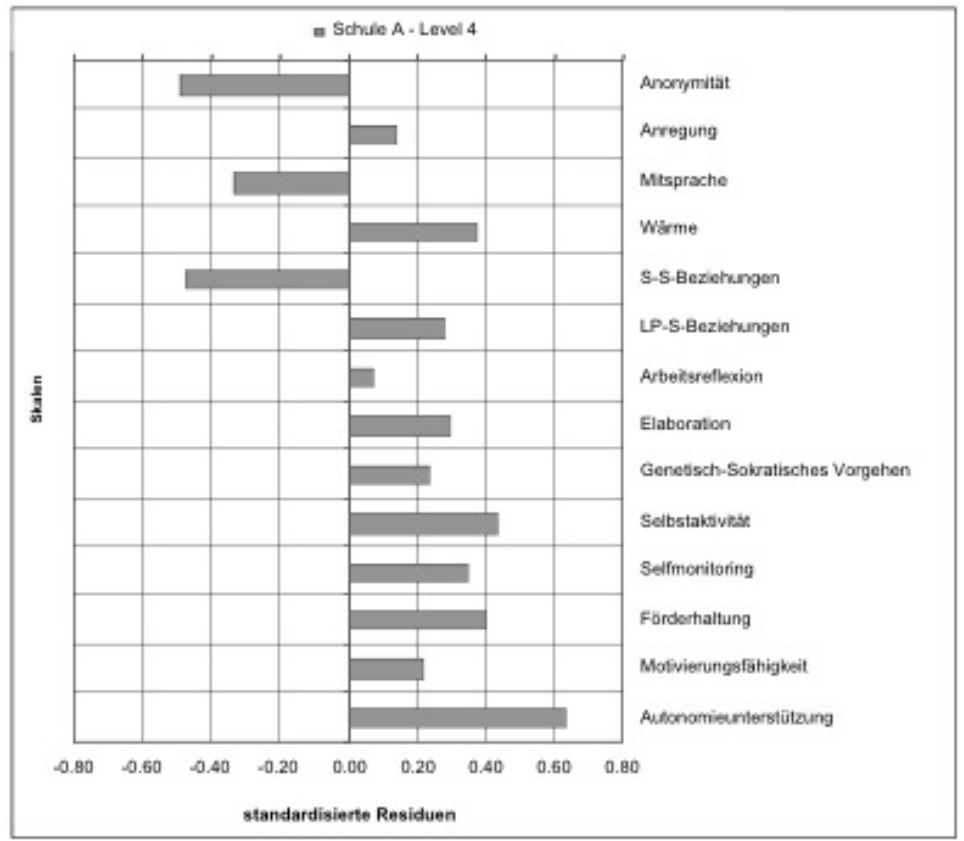

Abbildung 4: Profile der Level-3- und Level-4-Schule

\section{Diskussion}

Die Ergebnisse der in diesem Artikel bearbeiteten Fragestellungen, inwiefern sich die Gymnasien im Kanton Zürich im Ausmass der Implementation ausgewählter Prozessmerkmale von Teilautonomie systematisch unterscheiden und inwiefern sich diese Unterschiede in den Wahrnehmungen der Schüler/innen zu Schule und Unterricht abbilden, lassen sich folgendermassen zusammenfassen.

Entsprechend den Erwartungen können systematische Unterschiede zwischen den Schulen festgestellt werden. Die Level 4-Schule erreicht in praktisch allen Bereichen überdurchschnittliche Resultate. Eine hohe, fachbereichsübergreifende und differenzierte Kooperation zwischen den Lehrpersonen bildet sich 
auch bei den Schüler/innenwahrnehmungen in wichtigen schulischen Bereichen wie Unterrichtsgestaltung, Schul- und Klassenklima ab. Organisations-, Schulund Unterrichtsentwicklung stehen damit in einem positiven Zusammenhang zueinander. Dieses Resultat scheint stabil über fast alle untersuchten Bereiche zu sein. Im Gegensatz dazu erreichen die Schulen auf Level 1 und 2 fast durchgehend Resultate im unterdurchschnittlichen Bereich. Auch hier gibt es damit konsistente Befunde zwischen Organisations-, Schul- und Unterrichtsentwicklung, allerdings auf niedrigem Niveau.

Bei den Level-3-Schulen zeigt sich ein weniger konsistentes Bild. So zeigen die Schulen D und E zwar ein mit dem Kooperationsniveau kongruentes Bild in den wahrgenommenen Schul- und Unterrichtsqualitätsdimensionen. Eine relativ ausgeprägte wahrgenommene Kooperation zwischen den Lehrpersonen in verschiedenen Bereichen geht in diesen Schulen einher mit einem überdurchschnittlich positiv beurteilten Schul- und Klassenklima und einer selbstaktivierenden Unterrichtsorganisation. Die Abweichungen im positiven Bereich sind teilweise sogar grösser als bei der Level-4-Schule A. Hingegen schneiden die beiden anderen Level-3-Schulen $B$ und $C$ häufig unterhalb ihres Erwartungswertes ab, teilweise ana$\log$ zu den Ergebnissen der Level-1 und 2-Schulen. In diesen Schulen scheint es eine Diskrepanz zu geben zwischen der relativ ausgeprägten Kooperation zwischen den Lehrpersonen in der Schule, die von Seiten der Lehrpersonen wahrgenommen worden ist, und der eher geringen Unterrichts- und Schulqualität, wahrgenommen von Seiten der Schüler/innen. Organisations-, Schul- und Unterrichtsentwicklung sind damit nicht gleichermassen fortgeschritten. In weitergehenden Untersuchungen ist zu analysieren, ob strukturelle oder prozessuale Faktoren als Erklärung für dieses Resultat herangezogen werden können.

Entsprechend den Studien von Pang (2003), Cheung und Cheng (2002) und Kuper (2002) zeigen die Resultate dieser explorativen Studie, dass sich Schulen mit einem hohen Mass an dysfunktionalen oder funktionalen Prozessstrukturen identifizieren lassen. Die Schüler/innen in Schulen mit einem hohen Grad an Kooperation zwischen Lehrpersonen nehmen eine positivere Schul- und Unterrichtsgestaltung und mehr Unterstützung von ihren Lehrpersonen wahr als Schüler/innen in Schulen mit einem geringen Ausmass an Kooperation zwischen Lehrpersonen. Die diesbezügliche Trennlinie zwischen den Schulen verläuft zwischen den Niveaustufen 2 «Koordination» und 3 «Interaktion». Abweichend von diesem Ergebnismuster müssen zwar die beiden Level-3-Schulen $\mathrm{B}$ und $\mathrm{C}$ erwähnt werden. Werden allerdings pro Qualitätsdimension die durchschnittlichen Abweichungen aller Schulen auf Level 3 und 4 den durchschnittlichen Abweichungen aller Schulen auf Level 1 und 2 gegenübergestellt (ohne Darstellung), so wird dieses Ergebnismuster wiederum durchgehend bestätigt. Es scheint, dass sich erst eine relativ differenzierte und ausgeprägte Kooperation zwischen Lehrpersonen in einer hohen Schul- und Unterrichtsqualität abbildet und im unteren Bereich der Kooperationsausprägungen die verschiedenen $\mathrm{Ni}$ veaustufen weniger in der Schul- und Unterrichtsgestaltung differenzieren. 
Die Resultate dieser explorativen Studie unterstützen damit die These der Bedeutung der Prozessstuktur von teilautonomen Schulen für die Gestaltung eines optimalen Lernkontextes für die Schüler/innen. Es wird sichtbar, dass trotz identischer reglementarischer Vorgaben für alle Schulen eine deutliche Varianz zwischen den Schulen in der Umsetzung der Vorgaben vorzufinden ist, sobald die Prozessstrukturen der Schulen in den Analysen mitberücksichtigt werden. Weitergehende Analysen an einer grösseren Stichprobe haben insbesondere die Frage zu klären, ob der hier vorgefundene Zusammenhang in einem kausalen Verhältnis zu interpretieren ist oder ob zusätzliche Faktoren die Ergebnisse mitverursacht haben. Zudem ist das Instrumentarium von Steinert et al. (2006) dahingehend weiterzuentwickeln, dass wichtige Aspekte, die bis anhin nicht Bestandteil des Instrumentariums waren (z.B. Umgang mit Evaluationsergebnissen, Personalentwicklung), ins Instrumentarium aufgenommen werden.

\section{Literatur}

Avenarius, H., Kimmig, T. \& Rürup, M. (2003). Die rechtlichen Regelungen der Länder in der Bundesrepublik Deutschland zur erweiterten Selbstständigkeit der Schule. Eine Bestandesaufnahme. Frankfurt/Main: DIPF.

Baumert, J., Gruehn, S., Heyn, S., Köller, O. \& Schnabel, K. U. (1997). Bildungsverläufe und psychosoziale Entwicklung im Jugendalter (BIJU). Skalendokumentation. Berlin: MaxPlanck-Institut für Bildungsforschung.

Bortz, J. (1999). Statistik für Sozialwissenschaftler (5. Auflage). Berlin: Springer.

Burkard, C. \& Eikenbusch, G. (2002). Schulentwicklung international - eine Bilanz. Was können wir von den Erfahrungen anderer Länder lernen? Pädagogik, 54 (11), 44-49.

Cheung, F. W. M. \& Cheng, Y. C. (2002). An Outlier Study of Multilevel Self-Management and School Performance. School Effectiveness and School Improvement, 13 (3) , 253-290.

Clausen, M. (2002). Unterrichtsqualität: Eine Frage der Perspektive? Empirische Analysen zur Übereinstimmung, Konstrukt- und Kriteriumsvalidität (Bd. 29). Münster: Waxmann.

Dempster, N. (2000). Guilty or not: the impact and effects of site-based management on schools. Journal of Educational Administration, 38 (1), 47-63.

Ditton, H. (2000). Qualitätskontrolle und Qualitätssicherung in Schule und Unterricht. Ein Überblick zum Stand der empirischen Forschung. Zeitschrift für Pädagogik, 41. Beiheft, 73-92.

Eder, F. (1996). Das Schul- und Klassenklima. Innsbruck: Studien Verlag.

Eder, F. (1998). Linzer Fragebogen zum Schul-und Klassenklima (LFSK 8-13). Westermann test. Göttingen: Hogrefe.

Egloff, M. \& Zahner Rossier, C. (2005). Die Rolle der Schulen bei den Entscheidungsprozessen - Resultate aus der PISA-Studie und dem International Survey of Upper Secondary Schools (ISUSS) . Forschungsbeitrag am Jahreskongress der Schweizerischen Gesellschaft für Bildungsforschung (SGBF). Universität Lugano, 21. bis 23. September 2005.

Ekholm, M. (1997). Steuerungsmodelle für Schulen in Europa. Schwedische Erfahrungen mit alternativen Ordnungsmodellen. Zeitschrift für Pädagogik, 43 (4), 597-610.

Fend, H. (1977). Schulklima: Soziale Einflussprozesse in der Schule. Weinheim: Beltz.

Fend, H. (1986). «Gute Schulen - Schlechte Schulen». Die einzelne Schule als pädagogische Handlungseinheit. Deutsche Schule, 78 (3), 275-293.

Fend, H. (2004). Was stimmt mit den deutschen Bildungssystemen nicht? Wege zur Erklärung von Leistungsunterschieden zwischen Bildungssystemen. In G. Schümer, K.-J. Till- 
mann \& M. Weiss (Hrsg.), Die Institution Schule und die Lebenswelt der Schüler (S. 15-38). Wiesbaden: VS Verlag für Sozialwissenschaften.

Fend, H. (2005). Systemsteuerung im Bildungswesen - Anschlussfähigkeiten an die Schulwirklichkeit. In K. Maag Merki, A. Sandmeier, P. Schuler \& H. Fend (Hrsg.), Schule wohin? Schulentwicklung und Qualitätsmanagement im 21. Jahrhundert. Schriftenreihe zu «Bildungssystem und Humanentwicklung»: Berichte aus dem Forschungsbereich Schulqualität \& Schulentwicklung (S. 15-27). Forschungsbereich Schulqualität \& Schulentwicklung, Pädagogisches Institut, Universität Zürich.

Fend, H. (2006). Neue Theorie der Schule. Einführung in das Verstehen von Bildungssystemen. Lehrbuch. Wiesbaden: Verlag für Sozialwissenschaften.

Fend, H. \& Prester, H. G. (1986). Dokumentation der Skalen des Projektes Entwicklung im Jugendalter. Konstanz: Universität Konstanz.

Gruehn, S. (2000). Unterricht und schulisches Lernen. Münster: Waxmann.

Halbheer, U. \& Kunz, A. (2004). Profile von Zürcher Mittelschulen aufgrund "Pädagogischer EntwicklungsBilanzen" (PEB). Quantitative Analysen von Wahrnehmungen von Lehrpersonen. Unveröffentlichte Lizenziatsarbeit des Pädagogischen Instituts der Universität Zürich. Zürich.

Helmke, A. (2003). Unterrichtsqualität erfassen, bewerten, verbessern. Schulisches Qualitätsmanagement. Seelze/Velber: Kallmeyer.

Kanton Zürich (1999/2000). Mittelschulgesetz. Kanton Zürich: Regierungsrat.

Kotthoff, H.-G. (2005). Nicht-normative Steuerung in dezentralen Systemen. In J. Oebbeck (Hrsg.), Nicht-normative Steuerung in dezentralen Systemen (S. 231-251). Stuttgart: Franz Steiner Verlag.

Kuper, H. (2002). Entscheidungsstrukturen in Schulen. Eine differentielle Analyse der Schulorganisation. Zeitschrift für Pädagogik, 48 (6) , 856-878.

Leithwood, K. \& Menzies, T. (1998). Forms and effects of school-based management: A review. Educational Policy, 12 (3), 325-346.

Luyten, H., Visscher, A. J. \& Witziers, B. (2005). School Effectiveness Research: From a review of the critisism to recommendations for further development. School Effectiveness and School Improvement, 16 (3), 249-279.

Maag Merki, K. (Hrsg.). (2006). Lernort Gymnasium. Individuelle Entwicklungsverläufe und Schulerfahrungen. Bern: Haupt Verlag.

Moser, U., Keller, F. \& Tresch, S. (2002). Evaluation der 3. Primarschulklassen. Schlussbericht zuhanden der Bildungsdirektion des Kantons Zürich. Zürich: KBL, Universität Zürich.

OECD (1995). Decision-Making in 14 OECD Education Systems. Paris: OECD.

OECD (2004). Learning for Tomorrow's World - First Results from PISA 2003. Paris: OECD.

Pang, N. S.-K. (2003). Binding Forces and Teachers'School Life: A Recursive Model. School Effectiveness and School Improvement, 14 (3), 293-320.

Prenzel, M., Kristen, A., Dengler, P., Ettle, R. \& Beer, T. (1996). Selbstbestimmt motiviertes und interessiertes Lernen in der kaufmännischen Erstausbildung. Zeitschrift für Berufsund Wirtschaftspädagogik, Beiheft 13, 108-127.

Seashore, L. K. \& Leithwood, K. (1998). From organizational learning to professional learning communities. In K. Leithwood \& L. K. Seashore (Hrsg.), Organizational learning in schools (S. 275-285). Lisse: Swets en Zeitlinger.

Silins, H., Zarins, S. \& Mulford, B. (2002). What characteristics and processes define a school as learning organisation? Is this a useful concept to apply to schools? International Education Journal, 3 (1), 2432.

Steinert, B., Klieme, E., Maag Merki, K., Döbrich, P., Halbheer, U. \& Kunz, A. (2006). Lehrerkooperation in der Schule. Konzeption, Erfassung, Ergebnisse. Zeitschrift für Pädagogik, $52(2), 185-204$. 
Teddlie, C. \& Reynolds, D. (Hrsg.). (2001). The International Handbook of School Effectiveness Research. London: Routledge Falmer.

Watermann, R. \& Stanat, P. (2004). Schulrückmeldungen in PISA 2000: Sozialnorm- und kriteriumsorientierte Rückmeldeverfahren. Empirische Pädagogik, 18 (1), 40-61.

Wössmann, L. (2005). The Effect Heterogeneity of Central Examinations: Evidence from TIMSS, TIMSS-Repeat and PISA. Education Economics, 13 (2), 143-169.

Zahner Rossier, C., Berweger, S., Brühwiler, C., Holzer, T., Mariotta, M., Moser, U. et al. (2004). PISA 2003: Kompetenzen für die Zukunft. Erster nationaler Bericht. Neuchâtel, Bern: BFS/EDK.

Schlagworte: Lehrerkooperation, Schul- und Unterrichtsqualität, Schulische Teilautonomie, Gymnasium

\section{La structure des processus des écoles semi-autonomes et son efficacité de mise en oeuvre d'un contexte optimal pour apprendre}

\section{Résumé}

Les résultats obtenus lors d'études menées sur l'efficacité des écoles semi-autonomes sont non seulement inconsistants mais insuffisants. Nous manquons en particulier d'études réalisées sur un mode différencié et qui tiennent compte du degré de mise en application du système semi-autonome. La présente étude exploratrice porte sur la question de savoir dans quelle mesure le degré de mise en oeuvre de l'autonomie partielle dans les lycées se reflète dans la perception qu'ont les écoliers/ères de leur école et de leur enseignement. Les résultats obtenus permettent de constater que les écoliers/ères scolarisé(e)s dans des écoles où la coopération entre enseignants est systématiquement forte se sentent plus soutenus dans l'enseignement qui leur est prodigué - qu'ils perçoivent comme interactif et réfléchi - et ressentent le climat scolaire comme plus positif que les écoliers/ères scolarisé(e)s dans des établissements où la coopération entre enseignants est faible.

Mots clés: coopération entre enseignants, qualité de l'école et de l'enseignement, école semi-autonome, lycée 


\section{La struttura di processo delle scuole parzialmente autonome e la loro efficacia per la realizzazione di contesti d'apprendimento ottimali}

\section{Riassunto}

Le prove dell'efficacia delle scuole parzialmente autonome non sono soltanto incoerenti, ma anche insufficienti. In particolare mancano studi che analizzano le scuole parzialmente autonome in modo differenziato a seconda del loro grado di implementazione. Nel presente studio d'esplorazione si analizza l'entità in cui il grado di attuazione dell'autonomia parziale nei licei incida sulle percezioni degli studenti rispetto alla scuola e alle lezioni. I risultati mostrano che, nelle scuole con un elevato grado di cooperazione tra gli insegnanti, gli studenti percepiscono un clima scolastico positivo e una pianificazione delle lezioni con sostegno, riflessione e autonomia in misura sistematicamente crescente rispetto agli studenti in scuole con minor collaborazione tra gli insegnanti.

Parole chiave: cooperazione fra insegnanti, qualità dell'insegnamento e della scuola, autonomia della scuola, liceo

\section{The structure of the processes of semi-autonomous schools as a basis for an optimal context for learning}

\section{Summary}

Not only are the existing findings on the effectiveness of semi-autonomous schools inconsistent, these studies are also fundamentally insufficient. Lacking in particular are studies that differentiate among semi-autonomous schools according to degree to which autonomy has been put into practice. In the present explorative study, we pursue the question of whether the degree of autonomy implemented in academic-track secondary schools (Gymasien) is reflected in students' perceptions about school and classes. We find that in schools with a high level of cooperation among teachers, students perceive classes as more supportive, self-activating, and reflexive, and the school environment as more positive than in schools with a low level of cooperation among teachers.

Keywords: teacher cooperation, quality of schools, quality of teaching and learning, semi-autonomous school, high school 